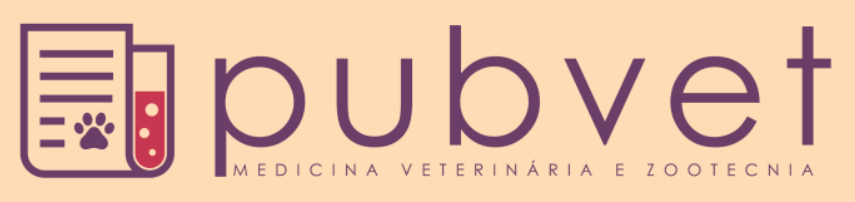

https://doi.org/10.22256/pubvet.v12n2a30.1-10

\title{
Craniectomia descompressiva de urgência para a sobrevivência de pacientes caninos vítimas de traumatismo crânio-encefálico grave
}

\author{
Ivone dos Santos Fraga ${ }^{1}$, Nuno Gonçalo Paixão Amaral dos Santos Almeida ${ }^{2}$, Allan \\ Andrade Rezende ${ }^{6}$ 3*
}

\author{
${ }^{I}$ Médica Veterinária Intensivista - Clínica Veterinária São Lázaro, Aracaju, Brasil \\ ${ }^{2}$ Médico Veterinário do Hospital Veterinário Central de Lisboa, Lisboa, Portugal. \\ ${ }^{3}$ Docente da Faculdade Pio Décimo, Aracaju, Brasil. \\ Autor para correspondência,E-mail: allan_a.rezende@hotmail.com
}

\begin{abstract}
RESUMO. A lesão cerebral causada pelo Trauma Crânio Encefálico tem duas componentes essenciais: a primária diz respeito à lesão mecânica causada pelas forças de aceleração, desaceleração, rotação e compressão as quais atuam sobre o crânio e cérebro no momento do impacto e a secundária que se refere a um número de alterações metabólicas e bioquímicas que se autoperpetuam e agravam o quadro inicial. A formação de hematomas e edema cerebral que levarão ao aumento da pressão intracraniana e compromisso da perfusão e oxigenação cerebrais. Quando não controlada a hipertensão intracraniana leva à hérnia cerebral e à morte do animal. A abordagem terapêutica à vítima de Trauma Crânio Encefálico e hipertensão intracraniana ainda é assunto de controversa pois não existem evidências clínicas e experimentais suficientes para elaborar um protocolo definitivo. É, no entanto, inquestionável que esta deverá ser expediente e agressiva.
\end{abstract}

Palavras chave: Traumatismo craniano, pressão intracraniana, sobrevida

\section{Emergency decompressive craniectomy for the survival of canine patients victims of severe cranio-encephalic trauma}

\begin{abstract}
The brain injury caused by the Brain Skull Trauma has two essential components: the primary one related to the mechanical injury caused by the forces of acceleration, deceleration, rotation and compression that act on the skull and brain at the moment of impact and the secondary one that refers to a number of metabolic and biochemical changes that self-perpetuate and aggravate the initial picture. The formation of hematomas and cerebral oedema can lead to an increase in intracranial pressure and compromise of cerebral perfusion and oxygenation. When uncontrolled, intracranial hypertension leads to cerebral herniation and death of the animal. The therapeutic approach to traumatic brain injury and intracranial hypertension is still controversial because there is insufficient clinical and experimental evidence to elaborate a definitive protocol. It is, however, unquestionable that this should be expedient and aggressive.
\end{abstract}

Keywords: Cranial trauma, intracranial pressure, survival

\section{Craniectomía descompresiva de urgencia para la supervivencia de pacientes caninos víctimas de traumatismo craneoencefálico grave}

RESUMEN. La lesión cerebral causada por el Trauma Cráneo Encefálico tiene dos componentes esenciales: la primaria que se refiere a la lesión mecánica causada por las fuerzas de aceleración, desaceleración, rotación y compresión que actúan sobre el cráneo y 
el cerebro en el momento del impacto y la secundaria que se refiere a un número de alteraciones metabólicas y bioquímicas que se auto perpetúan y agravan el cuadro inicial. La formación de hematomas y edema cerebral que llevarán al aumento de la presión intracraneal y compromiso de la perfusión y oxigenación cerebrales. Cuando no controlada la hipertensión intracraneal lleva a la hernia cerebral ya la muerte del animal. El abordaje terapéutico a la víctima de Trauma Cráneo Encefálico e hipertensión intracraneal sigue siendo algo controvertido, pues no existen evidencias clínicas y experimentales suficientes para elaborar un protocolo definitivo. Es, sin embargo, incuestionable que ésta deberá ser expediente y agresiva.

Palabras clave: Traumatismo craneal, presión intracraneal, sobrevida

\section{Introdução}

Há uma grande variedade de procedimentos cirúrgicos desenvolvidos com o intuito de tratar a hipertensão intracraniana de origem traumática (HIC). Nestes incluem-se a lobectomia temporal, a craniotomia circunferencial e várias técnicas de craniectomia descompressiva (CD) (Bullock et al., 2006). A remoção cirúrgica de uma porção do crânio quer por razões médicas ou supersticiosas é conhecida sob um contexto antropológico como trepanação. Evidências da realização deste tipo de craniectomia primitiva foram encontradas, espalhadas por todo o mundo, em esqueletos humanos tão antigos quanto 6.000 anos ( $\underline{\text { Schirmer }}$ et al., 2008).

O conceito moderno de craniectomia descompressiva para tratamento do Trauma Crânio Encefálico (TCE) em pacientes humanos foi elaborado em 1908 durante a $1^{\text {a }}$ Guerra Mundial. Ao aplicar a técnica de craniectomia descompressiva subtemporal às vítimas de guerra com traumatismo craniano, a qual já se usava desde 1905 para tratamento de hipertensão intracraniana associada a neoplasias (Mumert et al., 2012). Várias outras técnicas tanto uni como bilaterais foram desde então descritas. A hemicraniectomia que envolve a zona temporofrontoparietal e a craniectomia bifrontal são as mais populares e amplamente usadas (Qiu et al., 2009, Aarabi et al., 2009).

No contexto de TCE, a craniectomia está normalmente associada à evacuação de hematomas. Já o seu uso apenas como tratamento da hipertensão intracraniana é um assunto mais controverso. Porém o interesse nesta técnica é crescente e cada vez mais estudos em humanos são realizados com o objetivo de avaliar, muitos deles sugerem que a craniectomia descompressiva é eficaz na redução da pressão intracraniana (PIC) (Wani et al., 2009).
Vários dos dados disponíveis parecem também sugerir que o recurso à $\mathrm{CD}$ pode efetivamente resultar na redução da mortalidade e na contribuição para uma melhor recuperação funcional das vítimas de TCE. Todavia, a qualidade destes estudos não é suficiente para que se façam afirmações neste sentido (Taylor et al., 2001, Faleiro et al., 2005, Josan \& Sgouros, 2006, Aarabi et al., 2006, Timofeev et al., 2008, Qiu et al., 2009, Williams et al., 2009). Se na medicina humana o problema está na qualidade da informação existente devido à ausência de estudos prospectivos controlados e com alocação aleatória, já na Medicina Veterinária o maior problema está na ínfima quantidade de informação. Com a exceção de alguns estudos realizados por Bagley (1996), Schaller et al. (2003) e Chohan et al. (2013) não se tem sucesso em encontrar informações recentes sobre a eficácia da craniectomia descompressiva na redução da PIC bem como sobre os seus efeitos hemodinâmicos e metabólicos em cães ou gatos. $\mathrm{E}$ mesmo esta foi obtida a partir de animais clinicamente saudáveis. Isto equivale a dizer que, ao retirar algumas suposições Dewey (2006) e Chohan et al. (2013) não fora encontrado qualquer informação sobre o papel da craniectomia descompressiva no tratamento de cães gatos, vítimas de TCE. Assim, a grande maioria da informação que serviu como ponto de partida para o estudo retrospectivo aqui apresentado foi obtida de literatura médica humana.

A associação do TCE ao aumento da pressão intracraniana como resultado da formação de edema cerebral citotóxico e vasogênico, hematomas e contusões. As quais acarretam pressão intracraniana aumentada e levam à redução do fluxo sanguíneo cerebral e consequentemente à isquemia, na qual contribui para o agravamento e progressão da lesão secundária. Isto torna a $\mathrm{HIC}$ a responsável por uma grande parte das mortes nas vítimas de TCE. 
Com isto, o principal objetivo deste estudo retrospectivo foi o de avaliar qual a contribuição da craniectomia descompressiva de urgência para a sobrevivência de pacientes caninos vítimas de traumatismo crânio-encefálico grave.

\section{Material e Métodos}

\section{Pacientes}

Foram recolhidos os dados de pacientes admitidos no Hospital Veterinário Central de Lisboa - Lisboa no período compreendido entre 2003 e 2009. Definiu-se vítima de TCE grave como aquela que apresentava uma classificação na Escala de Coma de Glasgow (ECG) modificada igual ou inferior a 8 no momento da recepção, hiperglicemia e sinais evidentes de PIC aumentada (ausência de reflexo pupilar, presença de reflexo de Cushing, alteração do estado de consciência).

\section{Critérios de inclusão}

Com o objetivo de homogeneizar a amostra em estudo de forma a reduzir o número de variáveis foram definidos alguns critérios de inclusão. Todos os pacientes incluídos no estudo eram de espécie canina, tinham entre os 2 e os 8 anos de idade, entre os 10 e os $25 \mathrm{~kg}$ de peso, apresentavam história de TCE fechado grave, classificação na ECG à admissão igual ou inferior a 8 , pressão arterial sistólica entre os 80 e os 120 $\mathrm{mmHg}$ à admissão, níveis sanguíneos de glicose superiores a $200 \mathrm{mg} / \mathrm{dL}$ nas primeiras 4 horas pós- admissão, evidências de hipoventilação nas primeiras 4 horas pós-admissão, evidências de pressão intracraniana aumentada nas primeiras 4 horas pós-admissão, desenvolvimento de reflexo de Cushing nas primeiras 4 horas pós-admissão e evidências externas de TCE.

Para além dos pacientes que não possuíam as características acima referidas foram também excluídos todos aqueles que faleceram antes das 4 horas pós-admissão. No total, todos os que apresentavam outras lesões sistêmicas e orgânicas, não as resultantes do TCE, que pudessem pôr em risco a sua sobrevivência e todos aqueles cujo tratamento não foi iniciado antes das 3 horas póstraumatismo.

\section{Caracterização da amostra}

A amostra em estudo é composta por 86 indivíduos de raças caninas vítimas de TCE fechado grave, todos eles obedecem aos critérios de inclusão anteriormente expostos. As causas de traumatismo incluem atropelamentos (44), quedas (21) e violência (21). Os indivíduos foram submetidos a tratamento médico não tendo nenhum deles mostrado sinais de melhoria nas primeiras 4 horas pós-admissão. 40 (46,5\%) destes pacientes foram submetidos a craniectomia descompressiva de urgência após 4 horas de tratamento médico. Os restantes $46 \quad(53,5 \%)$ continuaram apenas com o tratamento médico. $\mathrm{Na}$ Tabela 1 são apresentadas as características de ambos os grupos.

Tabela 1. Características da população por grupo

\begin{tabular}{|c|c|c|c|}
\hline Parâmetro & Grupo com cirurgia (de estudo) & Grupo de controle & População total \\
\hline$\overline{\text { Número de observações }}$ & 40 & 46 & 86 \\
\hline Idade média \pm DP (Anos) & $4,63 \pm 1,64$ & $4,48 \pm 1,53$ & $4,55 \pm 1,58$ \\
\hline Peso médio \pm DP $(\mathrm{Kg})$ & $18,7 \pm 4,36$ & $18,26 \pm 4,55$ & $18,47 \pm 4,44$ \\
\hline Classificação ECG inicial média \pm DP & $7,5 \pm 0,64$ & $7,3 \pm 0,70$ & $7,4 \pm 0,67$ \\
\hline Glicose sanguíneo inicial média \pm DP $(\mathrm{mg} / \mathrm{dL}$ & $332 \pm 57$ & $333 \pm 63$ & $332 \pm 60$ \\
\hline Lactato sanguíneo inicial médio $\pm \mathrm{DP}(\mathrm{mmol} / \mathrm{L})$ & $7,16 \pm 1,69$ & $7,93 \pm 2,13$ & $7,57 \pm 1,97$ \\
\hline Hora de morte media \pm DP (horas) & $25,76 \pm 17,51$ & $20,45 \pm 5,54$ & $22,77 \pm 12,47$ \\
\hline \multicolumn{4}{|l|}{ Causa de traumatismo: } \\
\hline Atropelamento & 20 & 24 & 44 \\
\hline Queda & 11 & 10 & 21 \\
\hline Violência & 9 & 12 & 21 \\
\hline Pressão arterial sistólica inicial Média (mm/Hg) & $99,98 \pm 12,94$ & $97,06 \pm 12,32$ & $98,42 \pm 12,68$ \\
\hline
\end{tabular}

ECGm - Escala de coma de Glagow modificada; DP - Desvio padrão.

Tal como podemos ver na Tabela 1 , as populações de ambos os grupos apresentam características muito semelhantes, o que contribui para a confiabilidade dos resultados deste estudo. Esta suposição foi confirmada através da realização de um teste $\mathrm{t}$ de Student para 
comparação de médias, no qual, se verificou que não há diferenças significativas entre as idades, pesos, glicose e lactato sanguíneos iniciais e classificação ECG inicial dos dois grupos ( $\mathrm{p}=0.7$, $\mathrm{p}=0.65,0.97, \mathrm{p}=0.07, \mathrm{p}=0,18$ respectivamente) .

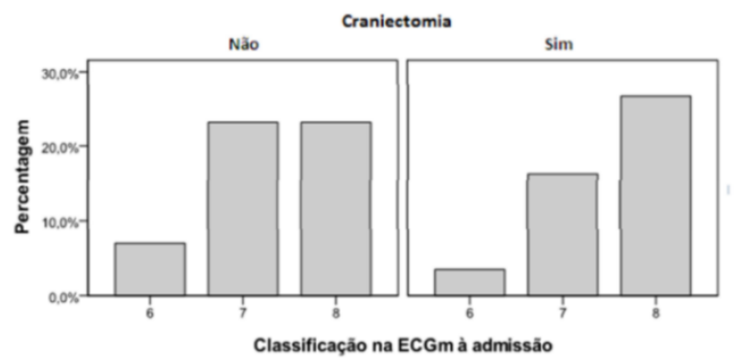

Figura 1. Gráfico das classificações da Escala de Coma de Glasgow em frequência relativa, por grupo. Nota-se que todos os indivíduos obtiveram uma classificação igual ou inferior a 8 , ou seja, todos eles foram vítimas de TCE considerado grave.

\section{Procedimento}

A abordagem inicial a todos os pacientes consistiu na realização de manobras de emergência de acordo com o $\mathrm{ABC}$ do trauma. Dado a todos os animais se encontrarem comatosos e com evidências de hipoventilação, procedeu-se de imediato à colocação de um tubo endotraqueal de forma a iniciar a ventilação assistida e a suplementação com oxigênio. A fluidoterapêutica foi iniciada de imediato de modo a corrigir ou evitar a hipotensão e a estabilizar as pressões sanguíneas entre os 100 e os $120 \mathrm{mmHg}$. Isso foi conseguido com recurso apenas a cristaloides (Lactato de Ringer ou solução salina a $0,9 \% 2,5-20 \mathrm{ml} / \mathrm{kg} / \mathrm{h}$ ). A analgesia inicial foi efetuada com recurso a morfina $(0,1-0,4 \mathrm{mg} / \mathrm{kg}$ IM) ou hidromorfona $(0,1-0,2 \mathrm{mg} / \mathrm{kg})$ e posteriormente mantida com fentanil $(2 \mu \mathrm{g} / \mathrm{kg} / \mathrm{h})$ ou hidromorfona $(12 \mu \mathrm{g} / \mathrm{kg} / \mathrm{h})$ em infusão contínua.

Após confirmação da existência de traumatismo craniano (anamnese, evidências externas) todos os pacientes foram posicionados de modo a manterem uma elevação da cabeça num ângulo de aproximadamente $30^{\circ}$ em relação ao plano horizontal, tendo-se o cuidado de não obstruir o fluxo sanguíneo jugular.

Todos os pacientes foram submetidos a uma avaliação neurológica inicial e à classificação através da Escala de Coma de Glasgow modificada em intervalos de 30 a 60 minutos. Assim que apresentavam sinais de hipertensão intracraniana iniciava-se a terapêutica hiperosmolar com recurso a manitol $(0,25 \mathrm{~g} / \mathrm{kg}$ administrado ao longo de $15 \mathrm{~min}$ e com um máximo de 2 administrações diárias) e solução salina hipertónica a 7,5\% (3 ml/kg com um máximo de 2 administrações diárias). Procedeu-se também à hiperventilação moderada de forma manual com o objetivo de manter os níveis de ETCO2 entre os 35 e os $45 \mathrm{mmHg}$.

Após quatro horas de tratamento sem resposta 40 dos 86 indivíduos foram submetidos a uma craniectomia descompressão de urgência, formando o grupo de estudo. Os restantes 46 continuaram apenas com o tratamento médico, formando assim o grupo de controle.

\section{Técnica cirúrgica}

Dado ao fato de todos os indivíduos se encontrarem comatosos no momento da cirurgia não foi necessária a utilização de anestesia para a realização do procedimento, tendo os animais suportado a intervenção apenas com recurso à analgesia. Não usar anestesia revelou-se vantajoso ao permitir uma reavaliação neurológica pósoperatória precisa imediatamente após a intervenção. A ventilação assistida foi mantida ao longo de todo o procedimento e monitorizada com recurso à capnografia e oximetria de pulso.

A decisão sobre em qual lado do crânio seria feita a descompressão foi tomada apenas com base nas lesões externas apresentadas pelos indivíduos. A craniectomia foi feita no lado que apresentava as lesões externas mais extensas assumindo-se que seria está a zona de golpe e que a probabilidade de edema e hematomas se desenvolverem nesta área seria maior, o que tornaria a descompressão mais eficaz. Após a cirurgia os pacientes retomaram a terapêutica hiperosmolar e a hiperventilação moderada sempre que os sinais de hipertensão intracraniana se mantiveram ou ressurgiram.

\section{Análise Estatística}

Os dados recolhidos foram analisados com recurso ao software de análise estatística SPSS Satistics 17.0. O teste t de Student foi usado para a comparação de médias das variáveis contínuas (Idade, Peso, classificação inicial na ECG, valores sanguíneos de glucose e lactato) e o teste de significância exacto de Fisher (two-tailed) para relacionar as duas variáveis categóricas (realização ou não de craniectomia com morte ou sobrevivência).

A análise de sobrevivência foi feita com recurso ao método de Kaplan-Meier. Os pacientes foram seguidos até às 72 horas pós-admissão ou 
até à morte dentro das 72 horas pós-admissão. A regressão de Cox foi usada para identificar o efeito de possíveis fatores de prognóstico no risco de morte nas primeiras 72 horas pós-admissão. Este procedimento foi levado a cabo na população total, comparando o grupo de estudo com o grupo de controlo, e utilizado para uma sub-análise dentro da população que realizou a craniectomia. Todos os resultados obtidos possuem um intervalo de confiança de $95 \%$,

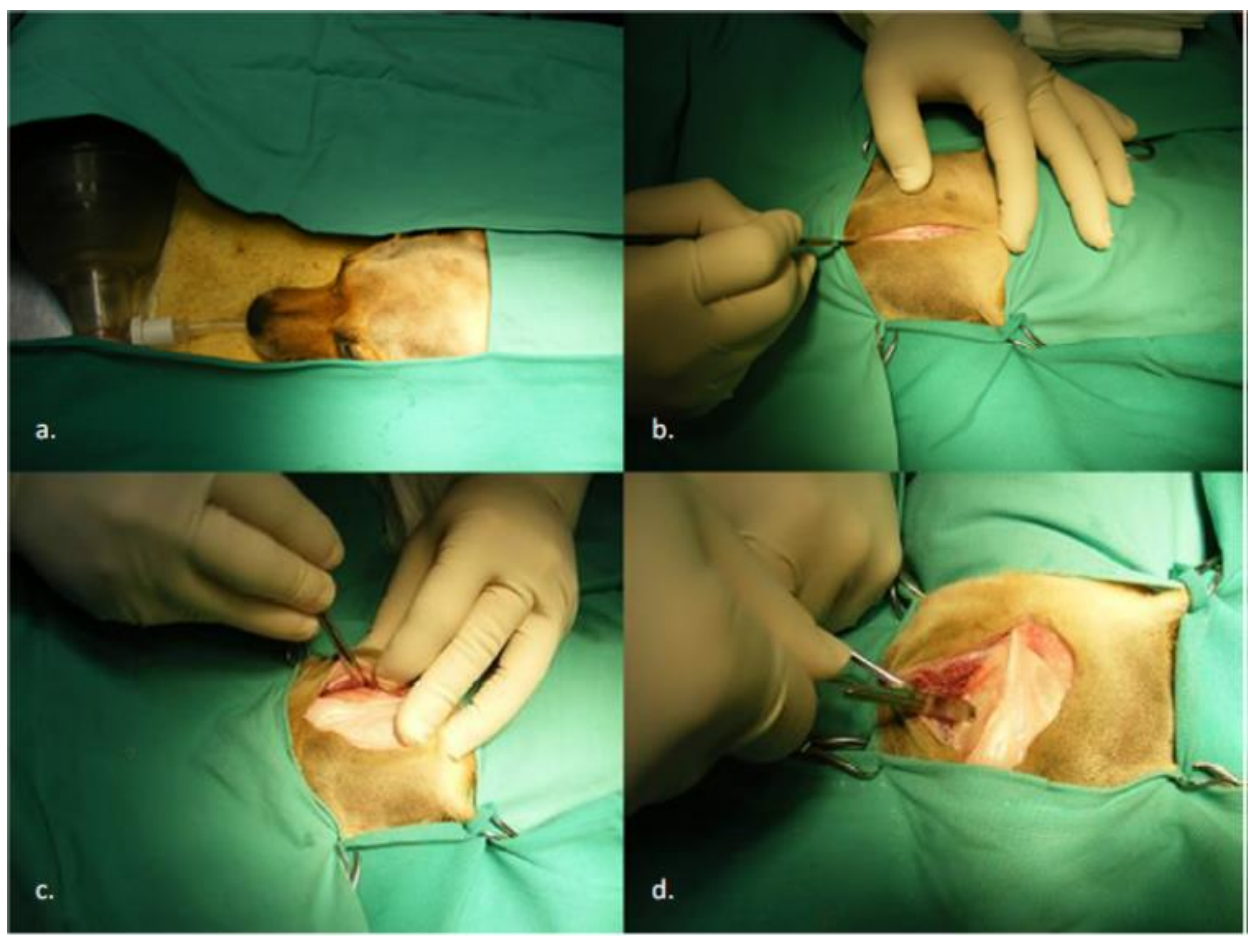

Figura 2. Craniectomia Descompressiva de Urgência: Técnica Cirúrgica I: a. Preparação do paciente; b. Incisão da pele; c. Rebatimento do musculo temporal; d. Craniotomia fronto-parietal com bisturi *Demonstração realizada em cadáver.

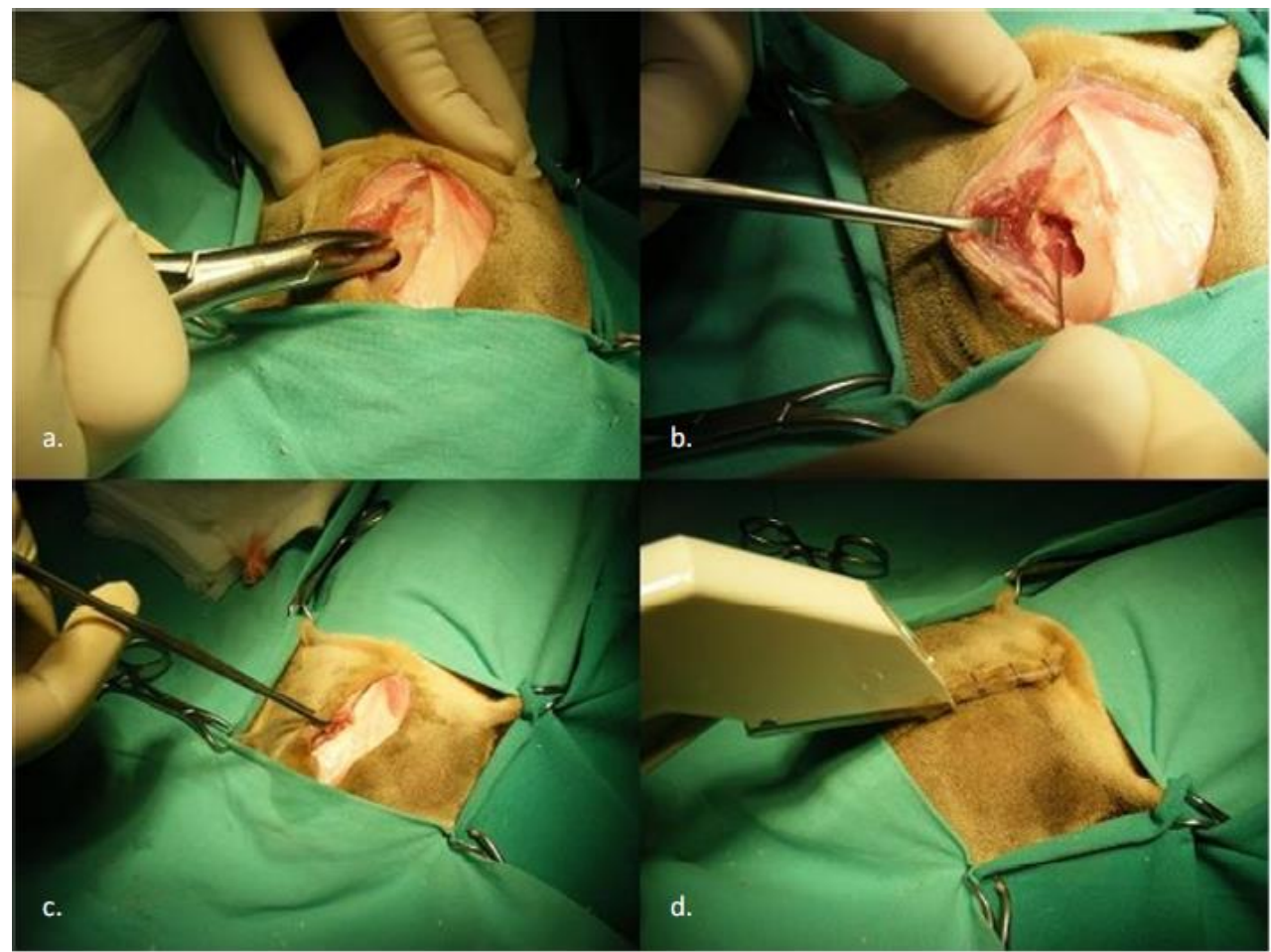

Figura 3. Craniectomia Descompressiva de Urgência: Técnica Cirúrgica II: a. Alargamento da janela com pinça goiva; b. Incisão das meninges com agulha $21 \mathrm{G}$; c. e d. Encerramento num único plano. *Demonstração realizada em cadáver. 


\section{Resultados}

Dos 86 indivíduos pertencentes à população inicial apenas 8 sobreviveram até às 72 horas pósadmissão, o que corresponde a uma taxa global de sobrevivência de $9,3 \%$ e uma taxa de mortalidade de $90,7 \%$.

Dos 8 sobreviventes 2 pertencem ao grupo de controlo e 6 ao grupo dos que realizaram craniectomia o que se traduz numa probabilidade acumulada de sobrevivência às 72 horas pósadmissão de, respectivamente, $4,3 \%$ e $15 \%$ (Figura 4).

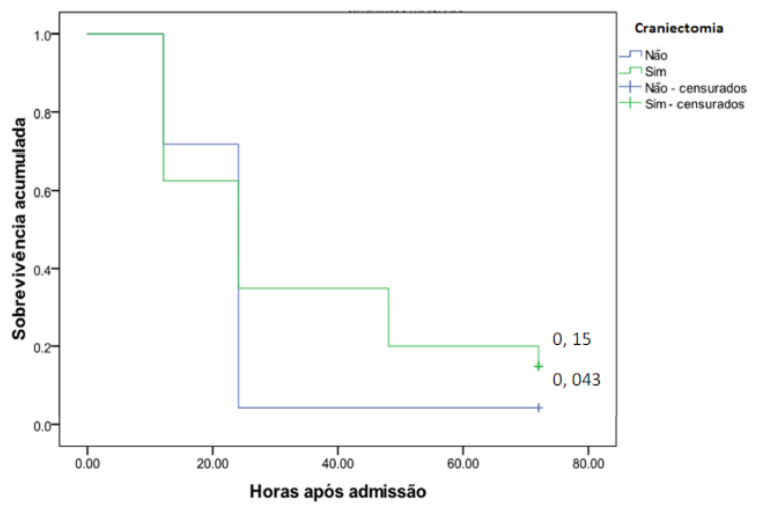

Figura 4. Gráfico de exposição da probabilidade acumulada de sobrevivência nos dois grupos (com e sem craniectomia) nas primeiras 72 horas pós admissão.

Verifica-se assim que a taxa de sobrevivência é cerca de 3,5 vezes superior no grupo que realizou a craniectomia. No entanto através do teste de exato de Fisher (2-tailed) não foi possível evidenciar uma relação estatisticamente significativa entre a realização de craniectomia descompressiva e a probabilidade de sobrevivência dos indivíduos.

Tabela 2. Testes de Chi-Quadrado e extrato de Fisher no qual expões a ausência de significado estático entre a realização de craniectomia e o aumento da taxa de sobrevivência.

\begin{tabular}{|c|c|c|c|c|}
\hline & Valor & $\begin{array}{l}\text { Graus de } \\
\text { liberdade }\end{array}$ & $\begin{array}{c}\mathrm{P} \\
\text { (2-tailed) }\end{array}$ & $\begin{array}{c}\mathrm{P} \\
\text { (1-tailed) }\end{array}$ \\
\hline $\begin{array}{l}\text { Chi Quadrado de } \\
\text { Pearson }\end{array}$ & 2,877 & 1 & 0,138 & 0,092 \\
\hline Teste exato de Fisher & & & 0,138 & 0,092 \\
\hline № de casos válidos & 86 & & & \\
\hline
\end{tabular}

É interessante notar que às 12 horas pósadmissão (8 horas pós-cirurgia) a taxa de sobrevivência acumulada chega mesmo a ser superior no grupo não cirúrgico $(71,7 \%$ contra os $62,5 \%$ do grupo da craniectomia).

O método para análise de sobrevivência de Kaplan-Meier mostra que as médias dos tempos de sobrevivência são de $22,7 \pm 1,7$ horas para o grupo de controlo (sem craniectomia) e de $32,7 \pm$ 3,7 para o grupo de teste (com craniectomia). No entanto nenhum dos testes para a igualdade de distribuições de sobrevivência (Log Rank, Breslow, Tarone-Ware) foi capaz de evidenciar diferenças estatisticamente significativas entre os dois grupos (Tabela 3).

Tabela 3. Testes de igualdade de distribuições de sobrevivência.

\begin{tabular}{lccc}
\hline & $\begin{array}{c}\text { Chi } \\
\text { Quadrado }\end{array}$ & $\begin{array}{c}\text { Graus de } \\
\text { liberdade }\end{array}$ & $\mathrm{P}$ \\
\hline Log Rank (Mantel-Cox) & 1,890 & 1 & 0,169 \\
$\begin{array}{l}\text { Breslow (Generalized } \\
\text { Wilcoxon) }\end{array}$ & 1,134 & 1 & 0,289 \\
Tarone-Ware & 1,693 & 1 & 0,193 \\
\hline
\end{tabular}

Foram analisados 4 possíveis fatores de prognóstico através da regressão de Cox, nomeadamente a idade dos indivíduos, a classificação na Escala de Coma de Glasgow modificada à admissão e os níveis sanguíneos iniciais de glicose e lactato. Porém não foram evidenciados quaisquer efeitos estatisticamente significativos sobre o risco de morte por parte de nenhum destes fatores (Tabela 4).

Tabela 4. Regressão de Cox (população total). ECG0: Classificação na escala de coma de Glasgow modificada à admissão.

\begin{tabular}{lccccc}
\hline & $\begin{array}{c}\text { Graus de } \\
\text { liberdade }\end{array}$ & $\mathrm{p}$ & \multirow{2}{*}{ Risco } & \multicolumn{2}{c}{ IC 95,0\% para risco } \\
\cline { 5 - 6 } & 1 & 0,731 & 1,022 & 0,887 & 1,179 \\
\hline Idade & 1 & 0,657 & 0,927 & 0,664 & 1,294 \\
ECG0 & 1 & 0,579 & 1,001 & 0,997 & 1,005 \\
Glucose & 1 & 0,078 & 1,106 & 0,989 & 1,237 \\
Lactato & 1 & & & & \\
\hline
\end{tabular}

O mesmo método foi utilizado para efetuar uma sub-análise ao considerar apenas os indivíduos que realizaram a craniectomia descompressiva. Neste estudo, o nível sanguíneo de lactato à admissão foi evidenciado como sendo um fator de risco com significado estatístico ( $\mathrm{p}<$ 0,05 , IC 95\%, 1,039 - 1,512), aumenta a probabilidade de morte em $25,3 \%$ por cada acréscimo de uma unidade no seu valor (Tabela 5). 
Tabela 5. Regressão de Cox (sub-análise no grupo de estudo). ECG0: Classificação na escala de coma de Glasgow modificada à admissão.

\begin{tabular}{lccccc}
\hline & $\begin{array}{c}\text { Graus de } \\
\text { liberdade }\end{array}$ & $\mathrm{p}$ & Risco & \multicolumn{2}{c}{ IC 95,0\% para risco } \\
\cline { 5 - 6 } & 1 & 0,374 & 1,110 & 0,882 & 1,395 \\
\hline Idade & 1 & 0,562 & 0,850 & 0,490 & 1,474 \\
ECG0 & 1 & 0,319 & 1,003 & 0,997 & 1,010 \\
Glucose & 1 & 0,018 & 1,253 & 1,039 & 1,512 \\
Lactato & 1 & &
\end{tabular}

Quatro dos 6 pacientes submetidos à craniectomia que sobreviveram desenvolveram abcessos que foram resolvidos facilmente com recurso à antibioterapêutica. Complicações mais frequentes neste tipo de cirurgia tais como pneumocéfalo ou hidrocefalia não foram verificadas Cavanaugh et al. (2008) e Yang et al. (2008). Não foi igualmente observada a ocorrência de hipotensão durante o procedimento cirúrgico (Miller et al., 2006)

\section{Discussão}

A craniectomia descompressiva é hoje usada no tratamento da hipertensão intracraniana refratária em vários centros neurocirúrgicos humanos como um último esforço na prevenção resultados desastrosos. Embora capaz de interromper a hipertensão incontrolável, a eficácia da

CD em melhorar a recuperação do paciente após TCE é questionável, o que se deve à ausência de evidências científicas com bases fortes (Aarabi et al., 2006).

\section{Redução da PIC}

Infelizmente durante a realização deste estudo, devido a limitações técnicas e econômicas, não foi possível monitorizar os valores da PIC dos vários indivíduos por forma a avaliar objetivamente a eficácia da CD na redução desses mesmos valores. A avaliação neurológica pós-cirúrgica poderia ser útil para averiguar este fato pela comparação de certos pontos tais como a resposta pupilar, as pressões sanguíneas e o nível de consciência dos pacientes com os seus equivalentes pré-cirúrgicos. No entanto, com grande pena do autor, este não teve acesso a esse dado visto os resultados póscirúrgicos mais precoces que lhe foram fornecidos corresponderem às 12 horas pós-admissão, ou seja, 8 horas após a cirurgia. Este intervalo é suficientemente grande para dar oportunidade à PIC de voltar a aumentar mesmo que tenha reduzido após a craniectomia. Todavia, a capacidade da craniectomia descompressiva em reduzir a PIC está bem documentada.

Chohan et al. (2013) verificaram, num estudo realizado em cães saudáveis, uma descida média dos valores de PIC de $2 \mathrm{mmHg}(\mathrm{p}<0,05)$ após craniectomia e de uns adicionais $7 \mathrm{mmHg}$ após durotomia ( $\mathrm{p}<0,01)$. Timofeev et al. (2008) verificaram resultados semelhantes com o mesmo procedimento realizado em gatos saudáveis. Observaram também que ocorria uma ligeira subida dos valores após encerramento da ferida cirúrgica, que ficavam, no entanto muito abaixo dos medidos inicialmente (média de $5.5 \mathrm{mmHg}$ contra os $10 \mathrm{mmHg}$ pré-cirúrgicos).

Estudos em humanos que provém a grande maioria da informação disponível. Segundo Harrington et al. (1996) pela da colocação de cateteres intraventriculares documentaram descidas dramáticas na PIC em vítimas de TCE grave e um aumento concomitante da complacência intracraniana, a qual foi testada através da injeção intracraniana de solução salina isotónica. Yoo et al. (1999) estudaram a pressão intraventricular em 20 pacientes com hipertensão intracraniana refratária que foram sujeitos a craniectomia frontotemporoparietal bilateral e verificaram uma redução da PIC em cerca de 50.2 $\pm 16.6 \%$ após o procedimento. Verificaram ainda uma redução adicional de $15.7 \pm 10.7 \%$ após incisão da dura-máter.

Polin et al. (2003) demonstraram haver uma redução significativa da PIC após craniectomia descompressiva bifrontal assim como uma diferença grande entre as PIC pós-operatórias e as PIC às 24 e 48 pós-trauma medidas em controlos tratados com coma barbitúrico retirados do Traumatic Coma Data Bank (TCDB).

Taylor et al. (2001) compararam o uso precoce da craniectomia bitemporal em adição ao tratamento médico intensivo com o recurso com apenas tratamento médico em 27 crianças com hipertensão intracraniana persistente. Verificaram uma descida em média de $8.98 \mathrm{mmHg}$ na PIC no grupo da craniectomia ao passo que no grupo de controlo esta foi de apenas $3.69 \mathrm{mmHg}$. 5 avaliaram 50 pacientes vítimas de TCE que foram sujeitos a craniectomia descompressiva, sem evacuação de lesões em massa, por apresentarem um aumento difuso do volume cerebral. Estes autores verificaram uma redução no valor médio de PIC de $24 \mathrm{mmHg}$ para $14.6 \mathrm{mmHg}$ e um aumento da PPC de uma média de $68.3 \mathrm{mmHg}$ para uma média de $73.3 \mathrm{mmHg}$. 
Josan \& Sgouros (2006) avaliaram 12 pacientes com PIC elevada após TCE que foram sujeitos a craniectomia frontotemporoparietal sem incisão da dura-máter e verificaram uma redução do valor médio de PIC de $30.66 \mathrm{mmHg}$ para 12.3 $\mathrm{mmHg}$. Num estudo semelhante em 27 pacientes. Timofeev et al. (2008) verificaram uma descida de $21.2 \mathrm{mmHg}$ para $15.7 \mathrm{mmHg}$ nos valores médios de PIC.

\section{Mortalidade, desfecho clínico e recuperação do paciente}

Tal como já foi apontado, este é um aspecto muito pouco ou mesmo nada estudado em medicina veterinária. Durante a pesquisa não foram encontrados nenhuns estudos com o objetivo de avaliar o impacto da craniectomia descompressiva na sobrevivência e recuperação de animais vítimas de TCE.

A atitude negativa de muitos clínicos e investigadores em relação à craniectomia descompressiva poderá ter origem nos resultados de estudos prematuros em humanos sobre o recurso à $\mathrm{CD}$ profilática, onde se obtiveram taxas de morbidade e mortalidade muito elevadas. Entre esses estudos encontra-se o de Cooper et al. (1979) que relatam uma série de 50 casos de pacientes submetidos a hemicraniectomia descompressiva para tratamento de hematoma subdural agudo.

Um objetivo comum a vários dos estudos realizados em medicina humana é a identificação de possíveis fatores de prognóstico, tal como discutido mais à frente. Também no presente estudo, apesar dos critérios de inclusão bastante apertados, se tentou fazer uma análise semelhante. A análise feita à população total não evidenciou a existência de nenhum fator de prognóstico. Todavia, em uma sub-análise feita aos 40 indivíduos que realizaram craniectomia, foi detectado que um acréscimo de 1 valor no nível de lactato sanguíneo à admissão está relacionado com um acréscimo de $25,3 \%$ na probabilidade de morte $(\mathrm{p}<0.05$, IC $95 \% 1,039-1,512)$. Isto sugere que este poderá ser um fator de prognóstico para cães vítimas de TCE que realizem craniectomia.

Notar que apenas se trata de uma sub-análise. Atualmente as taxas de mortalidade obtidas em estudos humanos são consideravelmente mais baixas, muito provavelmente devido aos avanços na medicina de urgência, nos cuidados intensivos e na tecnologia médico-cirúrgica assim como a uma seleção mais rigorosa das indicações para descompressão cirúrgica. Estudos recentes documentam taxas de mortalidade muito mais animadoras tais como 32\% (Williams et al., 2009), $28 \%$ (Aarabi et al., 2006) ou 15\% (Chibbaro et al., 2008). Josan \& Sgouros (2006) obtiveram a impressionante taxa de mortalidade de $0 \%$ em pacientes pediátricos o que vai de encontro às evidências de que pacientes mais jovens têm melhor prognóstico.

\section{Conclusões}

$\mathrm{O}$ presente estudo sugere que não existe relação entre a realização da craniectomia descompressiva de urgência e a probabilidade de sobrevivência às 72 horas pós-admissão de pacientes caninos vítimas de TCE grave fechado com hipertensão intracraniana refratária ao tratamento médico. Identificou também o nível sanguíneo de lactato à admissão como um possível fator de prognóstico para pacientes vítimas da patologia acima referida que realizem craniectomia descompressiva de urgência.

Detectou, no entanto, uma sugestão de que a craniectomia descompressiva possa ter alguma eficácia: esta produziu uma taxa de sobrevivência 3,5 vezes superior à do grupo de controle bem como um tempo médio de sobrevivência mais extenso.

\section{Referências Bibliográficas}

Aarabi, B., Hesdorffer, D. C., Ahn, E. S., Aresco, C., Scalea, T. M. \& Eisenberg, H. M. 2006. Outcome following decompressive craniectomy for malignant swelling due to severe head injury. Journal of Neurosurgery, 104, 469-479.

Aarabi, B., Hesdorffer, D. C., Simard, J. M., Ahn, E. S., Aresco, C., Eisenberg, H. M., McCunn, M. \& Scalea, T. 2009. Comparative study of decompressive craniectomy after mass lesion evacuation in severe head injury. Neurosurgery, 64, 927-940.

Bagley, R. S. 1996. Pathophysiologic sequelae of intracranial disease. Veterinary Clinics of North America: Small Animal Practice, 26, 711-733.

Bullock, M. R., Chesnut, R., Ghajar, J., Gordon, D., Hartl, R. \& Newell, D. W. 2006. Surgical management of traumatic brain injury. Neurosurgery, 58, S21 - S2-62.

Cavanaugh, R. P., Aiken, S. W. \& Schatzberg, S. J. 2008. Intraventricular tension pneumocephalus and cervical subarachnoid 
pneumorrhachis in a bull mastiff dog after craniotomy. Journal of Small Animal Practice, $49,244-248$.

Chibbaro, S., Marsella, M., Romano, A., Ippolito, S. \& Benericetti, E. 2008. Combined internal uncusectomy and decompressive craniectomy for the treatment of severe closed head injury: experience with 80 cases. Journal of Neurosurgery, 108, 74-79.

Chohan, A. S., Greene, S. A., Keegan, R. D., Grubb, T. L. \& Chen, A. V. 2013. Intracranial pressure and cardiopulmonary variables during isoflurane or sevoflurane anesthesia at various minimum alveolar concentration multiples in normocapnic dogs. American Journal of Veterinary Research, 74, 369-374.

Cooper, P. R., Hagler, H., Clark, K. W. \& Barnett, P. 1979. Enhancement of experimental cerebral edema after decompressive craniectomy: implications for the management of severe head injuries. Neurosurgery, 4, 296300 .

Dewey, C. W. 2006. Encefalopatias: distúrbios cerebrais. Roca, São Paulo.

Faleiro, R. M., Pimenta, N. J. G., Faleiro, L. C. M., Cordeiro, A. F., Maciel, C. J. \& Gusmão, S. N. S. 2005. Craniotomia descompressiva para tratamento precoce da hipertensão intracraniana traumática. Arquivo Neuropsiquiatrico, 63, 508-513.

Harrington, M. L., Bagley, R. S., Moore, M. P. \& Tyler, J. W. 1996. Effect of craniectomy, durotomy, and wound closure on intracranial pressure in healthy cats. American Journal of Veterinary Research, 57, 1659-1661.

Josan, V. A. \& Sgouros, S. 2006. Early decompressive craniectomy may be effective in the treatment of refractory intracranial hypertension after traumatic brain injury. Child's Nervous System, 22, 1268-1274.

Miller, P., Mack, C. D., Sammer, M., Rozet, I., Lee, L. A., Muangman, S., Wang, M., Hollingworth, W., Lam, A. M. \& Vavilala, M. S. 2006. The incidence and risk factors for hypotension during emergent decompressive craniotomy in children with traumatic brain injury. Anesthesia \& Analgesia, 103, 869-875.

Mumert, M. L., Altay, T. \& Couldwell, W. T. 2012. Technique for decompressive craniectomy using Seprafilm as a dural substitute and anti-adhesion barrier. Journal of Clinical Neuroscience, 19, 455-457.

Polin, R. S., Ayad, M. \& Jane, J. A. 2003. Decompressive craniectomy in pediatric patients. Critical Care, 7, 409-411.

Qiu, W., Guo, C., Shen, H., Chen, K., Wen, L., Huang, H., Ding, M., Sun, L., Jiang, Q. \& Wang, W. 2009. Effects of unilateral decompressive craniectomy on patients with unilateral acute post-traumatic brain swelling after severe traumatic brain injury. Critical Care, 13, R185.

Schaller, B., Graf, R., Sanada, Y., Rosner, G., Wienhard, K. \& Heiss, W.-D. 2003. Hemodynamic and metabolic effects of decompressive hemicraniectomy in normal brain: An experimental PET-study in cats. Brain Research, 982, 31-37.

Schirmer, C. M., Ackil, A. A. \& Malek, A. M. 2008. Decompressive craniectomy. Neurocritical Care, 8, 456-470.

Taylor, A., Butt, W., Rosenfeld, J., Shann, F., Ditchfield, M., Lewis, E., Klug, G., Wallace, D., Henning, R. \& Tibballs, J. 2001. A randomized trial of very early decompressive craniectomy in children with traumatic brain injury and sustained intracranial hypertension. Child's Nervous System, 17, 154-162.

Timofeev, I., Czosnyka, M., Nortje, J., Smielewski, P., Kirkpatrick, P., Gupta, A. \& Hutchinson, P. 2008. Effect of decompressive craniectomy on intracranial pressure and cerebrospinal compensation following traumatic brain injury. Journal of Neurosurgery, 108, 66-73.

Wani, A. A., Dar, T. I., Ramzan, A. U., Malik, N. K., Kirmani, A. R., Bhatt, A. R., Chhiber, S. S., Javaid, S. \& Wani, M. A. 2009. Decompressive craniectomy in head injury. The Indian Journal of Neurotrauma, 6, 103-110.

Williams, R. F., Magnotti, L. J., Croce, M. A., Hargraves, B. B., Fischer, P. E., Schroeppel, T. J., Zarzaur, B. L., Muhlbauer, M., Timmons, S. D. \& Fabian, T. C. 2009. Impact of decompressive craniectomy on functional outcome after severe traumatic brain injury. Journal of Trauma and Acute Care Surgery, 66, 1570-1576.

Yang, X. F., Wen, L., Shen, F., Li, G., Lou, R., Liu, W. G. \& Zhan, R. Y. 2008. Surgical complications secondary to decompressive 
craniectomy in patients with a head injury: a series of 108 consecutive cases. Acta Neurochirurgica, 150, 1241.

Yoo, D.-S., Kim, D.-S., Cho, K.-S., Huh, P.-W., Park, C.-K. \& Kang, J.-K. 1999. Ventricular pressure monitoring during bilateral decompression with dural expansion. Journal of Neurosurgery, 91, 953-959.
Article History:

Received 12 October 2017

Accepted 02 November 2017

Available online 15 January 2018

License information: This is an open-access article distributed under the terms of the Creative Commons Attribution License 4.0, which permits unrestricted use, distribution, and reproduction in any medium, provided the original work is properly cited. 